\title{
Safety and Efficacy of the Noncessation Method of Antithrombotic Agents after Emergency Endoscopic Hemostasis in Patients with Nonvariceal Upper Gastrointestinal Bleeding: A Multicenter Pilot Study
}

\author{
Daisuke Yamaguchi $\mathbb{D}^{1,2}$ Naoyuki Tominaga, ${ }^{3}$ Koichi Miyahara, ${ }^{4}$ Nanae Tsuruoka, \\ Yasuhisa Sakata, ${ }^{2}$ Yuki Takeuchi, ${ }^{1}$ Takuya Matsunaga, ${ }^{3}$ Hidenori Hidaka, ${ }^{4}$ \\ Takashi Akutagawa, ${ }^{2}$ Takahiro Noda, ${ }^{4}$ Shinichi Ogata, ${ }^{3}$ Seiji Tsunada, ${ }^{1}$ \\ and Motohiro Esaki ${ }^{2}$ \\ ${ }^{1}$ Department of Gastroenterology, National Hospital Organization Ureshino Medical Center, Ureshino, Japan \\ ${ }^{2}$ Division of Gastroenterology, Department of Internal Medicine, Faculty of Medicine, Saga University, Saga, Japan \\ ${ }^{3}$ Department of Gastroenterology, Saga-Ken Medical Centre Koseikan, Saga, Japan \\ ${ }^{4}$ Department of Internal Medicine, Karatsu Red Cross Hospital, Karatsu, Japan
}

Correspondence should be addressed to Daisuke Yamaguchi; daisukehawks@gmail.com

Received 6 December 2020; Revised 1 May 2021; Accepted 13 May 2021; Published 22 May 2021

Academic Editor: Laura Turco

Copyright (c) 2021 Daisuke Yamaguchi et al. This is an open access article distributed under the Creative Commons Attribution License, which permits unrestricted use, distribution, and reproduction in any medium, provided the original work is properly cited.

\begin{abstract}
Background and Aims. The present study aimed to clarify the safety and efficacy of the noncessation method of antithrombotic agents after emergency endoscopic hemostasis in patients with nonvariceal upper gastrointestinal bleeding (UGIB). Methods. In this multicenter, prospective, pilot study, we performed emergency endoscopic hemostasis for nonvariceal UGIB in patients taking antithrombotic agents and resumed the medications without a cessation period (group A). The clinical characteristics, types of antithrombotic agents, UGIB etiology, treatment outcome, and adverse events were evaluated. We used propensity score matching to compare treatment outcomes and adverse events with our previous cohort (group B) in whom antithrombotic agents were transiently discontinued after emergency endoscopic hemostasis. Results. Forty-three consecutive patients were prospectively enrolled. The main antithrombotic agents were low-dose aspirin and direct oral anticoagulants; 11 patients (25.6\%) were taking multiple antithrombotics. Peptic ulcers were the main cause of bleeding (95.4\%). Endoscopic hemostasis was successful in all patients and the incidence of rebleeding within a month was $7.0 \%$. Propensity score matching created 40 matched pairs. Endoscopic hemostasis was performed by soft coagulation significantly more frequently in group A than in group B (97.5\% versus $60.0 \%, P<0.001)$. Neither the rebleeding rate within a month nor thromboembolic event rate was different between the two groups. However, the mean duration of hospitalization was significantly shorter in group A than in group B $(8.6 \pm 5.2 \mathrm{~d}$ versus $14.4 \pm 7.1 \mathrm{~d}, P<0.001)$. Conclusions. Antithrombotic agents possibly can be continued after successful emergency endoscopic hemostasis for nonvariceal UGIB.
\end{abstract}

\section{Introduction}

Nonvariceal upper gastrointestinal bleeding (UGIB) is one of the most common causes of gastrointestinal emergencies, sometimes requiring emergency endoscopic hemostasis [1-7]. This is especially the case with antithrombotic agents
[8-10], and such patients usually exhibit more severe clinical signs of nonvariceal UGIB [11]. It therefore seems important to determine optimal clinical management of nonvariceal UGIB, especially in patients requiring emergency endoscopic hemostasis under antithrombotic treatment. Theoretically, antithrombotic agents should be stopped after 
endoscopic hemostasis considering the risk of recurrent UGIB. However, cessation of antithrombotic agents exposes the patients to the risk of thromboembolic events because of their underlying condition [12]. Such thromboembolic events can occur even in patients having only 5-7 days' discontinuation of the medications [13]. Considering these conflicting risks, the Japan Gastroenterological Endoscopy Society (JGES) revised the endoscopic management guidelines in 2012, which focused more on avoiding thromboembolic events than on GI bleeding adverse events [14]. We previously compared the clinical management of patients with nonvariceal UGIB before and after the revision of the JGES guideline [15] and demonstrated that the cessation period of antithrombotic agents was significantly shortened after the revision of the guideline [11]. However, the risk of rebleeding after emergency endoscopic hemostasis without a cessation period remains uncertain.

The present study aimed (1) to evaluate the safety of the noncessation method of antithrombotic agents after emergency endoscopic hemostasis in patients with nonvariceal UGIB and (2) to compare the clinical outcome and adverse events with our previous cohort in whom antithrombotic agents were transiently discontinued after emergency endoscopic hemostasis.

\section{Methods}

2.1. Study Design and Ethical Issues. This was designed as a multicenter prospective pilot study. Patients who had undergone emergency endoscopy and endoscopic hemostasis for nonvariceal UGIB from February 2018 through October 2019 at participating institutions were enrolled. Patientsabove 20 years of age who fulfilled the following criteria were candidates for the study: (1) underwent endoscopic hemostasis within $24 \mathrm{~h}$ of symptom onset; (2) used antithrombotic agents, including antiplatelet agents and anticoagulants; and (3) had a clear level of consciousness as well as stable respiratory and circulatory dynamics. Patients with variceal bleeding were excluded. Written informed consent was obtained from patients who met the inclusion criteria after endoscopic hemostasis.

This study was conducted in accordance with the Declaration of Helsinki and the guidelines of the Consolidated Standards of Reporting Trials (CONSORT). The study protocol and the consent procedure were approved by the Ethics Review Committee of the National Hospital Organization Ureshino Medical Center (approval number 17-24) and registered with the University Hospital Medical Information Network (UMIN) Clinical Trials Registry (UMIN000030879) on 22 January 2018.

2.2. Patient Data. Clinical data collected in the prospective cohort were age, gender, alcohol consumption, smoking habit, H. pylori infection, hemoglobin level, comorbidity (including Charlson comorbidity score), and types of medications at study entry (antithrombotic agents, nonsteroidal anti-inflammatory drugs, and antacids). $H$. pylori infection was diagnosed by the serum levels of anti- $H$. pylori antibodies, the urea breath test, or the rapid urease test. We also accumulated information concerning endoscopic findings, hemostatic procedure, and treatment outcome (transfusion volume, fasting period, and hospitalization period). All patients in the prospective cohort resumed antithrombotic agents after emergency endoscopic hemostasis without a cessation period (group A).

In the present study, we used the clinical data of our retrospective cohort of 154 patients on antithrombotic treatment who underwent emergency endoscopic hemostasis for nonvariceal UGIB from January 2002 through December 2013 at Saga Medical University Hospital as group B (Supplementary Tables 1-3) [11]. These patients were similarly treated but antithrombotic agents were transiently discontinued after endoscopic hemostasis in accordance with the JGES guideline at that time $[12,13]$.

2.3. Endoscopic Hemostasis. Endoscopic hemostasis was performed mainly by high-frequency soft coagulation or hemoclipping using a forward-viewing water-jet endoscope (GIF-Q260J; Olympus, Tokyo, Japan) [16, 17]. The choice of procedure was at the endoscopists' discretion. Soft coagulation was performed using an electrosurgical unit (ICC 200, ERBE Elektronedizin GmbH, Tübingen, Germany) and monopolar hemostatic forceps (Radial Jaw ${ }^{\mathrm{TM}} 4$ Hot Biopsy Forceps, Boston Scientific, Tokyo, Japan) inserted through the channel of the endoscope [16]. Briefly, when the bleeding vessel was identified, electric current was gently applied using the closed forceps to the area surrounding the target vessel using the soft coagulation mode $(60 \mathrm{~W})$. Once blood flow diminished, the endoscopist coagulated the target vessel using $80 \mathrm{~W}$ in gastric ulcers and $60 \mathrm{~W}$ in duodenal ulcers [16]. Hemoclipping was performed using hemoclips (HX610-135, HX-610-135S hemoclips; Olympus Optical, Tokyo, Japan) that were placed on the ulcer base to bind the detected vessels $[16,18]$. In both procedures, when the ulcer base was covered by massive clots, the clots were initially removed either by water jet or by endoscopic forceps to visualize the vessel(s) responsible. Both procedures were repeated until hemostasis was endoscopically confirmed. Interventional radiology and/or surgery was implemented when the endoscopic hemostasis was considered ineffective.

After endoscopic hemostasis was achieved, all patients were hospitalized and managed conventionally (fasting with peripheral parenteral nutrition and intravenous proton pump inhibitors). The indications for blood transfusion were a hemoglobin level $<6 \mathrm{~g} / \mathrm{dL}$ on admission or a rapid drop in hemoglobin level $>2 \mathrm{~g} / \mathrm{dL}$ in patients with hemoglobin level $<10 \mathrm{~g} / \mathrm{dL}$ at baseline. Follow-up endoscopy was performed on patients who were judged necessary by the endoscopist 48-72 $\mathrm{h}$ after the initial endoscopy, and repeat endoscopic hemostasis was applied in case recurrent UGIB was detected. Interventional radiology and/or surgery was implemented when the endoscopic hemostasis was considered ineffective. After resumption of oral nutrition, oral administration of the proton pump inhibitors was performed for at least a month in both groups. 
All endoscopic hemostasis was performed by 18 endoscopists, comprising 8 specialists and 10 trainees. Specialists were defined as endoscopists who had performed endoscopy for more than 5 years with experience in more than 40 endoscopic submucosal dissection (ESD) procedures after mastering the required fundamental skills and knowledge $[19,20]$.

2.4. Study Outcomes. The primary endpoint of the present study was the rate of rebleeding within a month after emergency endoscopic hemostasis in group A. Rebleeding was defined as follows: (1) follow-up endoscopy identified recurrent UGIB or the stigmata of recent hemorrhage or (2) melena and progressive anemia with a decrease in hemoglobin level greater than $2 \mathrm{~g} / \mathrm{dL}$ and/or with a decrease in systolic blood pressure $<80 \mathrm{mmHg}$. The secondary endpoint was the incidence of adverse events within a month (rebleeding, thromboembolic events, and mortality) in group A.

To compare the noninferiority of the noncessation method of antithrombotic agents to the transient discontinuation method based on the JGES guidelines, treatment outcome and adverse events in group A were compared with those in group B. Group B was the retrospective cohort of 154 patients with nonvariceal UGIB who underwent emergency endoscopic hemostasis and in whom antithrombotic agents were subsequently discontinued [11].

2.5. Sample Size Calculation and Statistical Analysis. The sample size of the noninferiority study was calculated based on the rate of rebleeding within a month. The rebleeding rate after emergency endoscopic hemostasis in patients with nonvariceal UGIB taking antithrombotic agents was 9.7\% (15/154 patients) in our previous study [11]. We also considered other studies that described bleeding rates after gastric ESD among patients on antithrombotic treatments (7.0\%-21.1\%) [21-23]. Based on these results, we assumed the overall rebleeding rate in group A to be $20 \%$. We hypothesized that a difference of $10 \%$ or less (prespecified noninferiority margin for this endpoint) in the rebleeding rate between the two groups would constitute a clinically meaningful difference. Assuming a power of $80 \%$ and an alpha of 0.05 (one-sided), at least 78 patients ( 39 patients in each group) would be required in the noninferiority study. Assuming that $10 \%$ of patients could be lost to follow-up, a sample size of 86 patients (43 patients in each group) was planned. The rebleeding rate in group A would be considered noninferior to that in group B if the $95 \%$ confidence interval (CI) for the treatment difference was greater than $-10.0 \%$.

Treatment outcome and adverse events were compared between groups A and B using propensity score matching analysis. This method was applied to adjust significant differences in the baseline characteristics of the patients and reduce the influence of possible confounding factors [24]. The two groups were matched at a 1:1 ratio (40 patients in each group) with the adjustment for nine covariates (age, gender, $H$. pylori infection, number of agents
TABLE 1: Characteristics of patients in group $\mathrm{A}^{*}$.

\begin{tabular}{lc}
\hline Number of patients $(\mathrm{N})$ & 43 \\
\hline Age (years) & $77.8 \pm 9.1$ \\
\hline Gender, males & $28(65.1 \%)$ \\
\hline Alcohol drinking & $6(13.9 \%)$ \\
\hline Smoking & $16(37.2 \%)$ \\
\hline Helicobacter pylori infection & $20(46.5 \%)$ \\
\hline Using NSAIDs & $10(23.2 \%)$ \\
\hline Using gastric acid secretion inhibitor & $11(25.5 \%)$ \\
\hline Comorbidity & $23(53.5 \%)$ \\
Cardiovascular diseases & $14(32.6 \%)$ \\
Cerebrovascular diseases & $8(18.6 \%)$ \\
Chronic kidney diseases & $2(4.6 \%)$ \\
Chronic liver damage & $14(32.6 \%)$ \\
Diabetes mellitus & $32(74.4 \%)$ \\
Hypertension & $10(23.3 \%)$ \\
Malignant diseases & $1.8 \pm 1.0$ \\
\hline Charlson comorbidity score
\end{tabular}

${ }^{*}$ Results are presented as number of patients or mean \pm standard deviation. Group A: patients taking antithrombotic agents who underwent emergency endoscopic hemostasis for nonvariceal upper gastrointestinal bleeding and resumed antithrombotic medication without a cessation period; NSAIDs: nonsteroidal anti-inflammatory drugs.

TABle 2: Types of antithrombotic agents taken by patients in group A.

\begin{tabular}{lc}
\hline Number of antithrombotic agents & 43 \\
Single agent & $32(74.4 \%)$ \\
Multiple agents & $11(25.6 \%)$ \\
\hline Types of antithrombotic agents & \\
Antiplatelet agents & $17(39.5 \%)$ \\
Aspirin & $11(25.6 \%)$ \\
Clopidogrel & $3(7.0 \%)$ \\
Sarpogrelate & $2(4.6 \%)$ \\
Prostaglandin preparations & $2(4.6 \%)$ \\
Prasugrel & $1(2.3 \%)$ \\
Cilostazol & \\
\hline Anticoagulant agents & $12(27.9 \%)$ \\
DOACs & $6(14.0 \%)$ \\
Edoxaban & $4(9.3 \%)$ \\
Apixaban & $2(4.6 \%)$ \\
Rivaroxaban & $5(11.6 \%)$ \\
Warfarin &
\end{tabular}

DOACs: direct oral anticoagulants. Group A: patients taking antithrombotic agents who underwent emergency endoscopic hemostasis for nonvariceal upper gastrointestinal bleeding and resumed antithrombotic medication without a cessation period.

[antithrombotic, antiplatelet, and anticoagulant], and ulcer characteristics [location, number, and size]) to minimize inherent bias. These nine covariates were selected based on the opinions of expert endoscopists (DY, NT, KM, TN, SO, and ST). This model yielded a C statistic of 0.705 , indicating preferable ability for the comparison between groups $\mathrm{A}$ and B. The caliper width of propensity score matching was 0.20 .

Categorical data were expressed as a number (percentage), and the chi-square test was used to identify differences between the two groups. Numerical data for a normal distribution were expressed as the mean \pm the standard 
TABLE 3: Endoscopic findings and hemostatic methods in patients in group $\mathrm{A}$.

\begin{tabular}{lc}
\hline Peptic ulcer & $41(95.4 \%)$ \\
Angioectasia & $2(4.6 \%)$ \\
Location of ulcer & \\
Gastric ulcer & $34(79.1 \%)$ \\
$\quad$ Upper third & $5(11.6 \%)$ \\
$\quad$ Middle third & $23(53.5 \%)$ \\
$\quad$ Lower third & $6(13.9 \%)$ \\
Duodenal ulcer & $7(16.3 \%)$ \\
Number of ulcers & \\
Single & $26(60.5 \%)$ \\
Multiple & $15(34.9 \%)$ \\
Size of ulcer (mm) & \\
0-10 & $20(46.5 \%)$ \\
$>11$ & $21(48.1 \%)$ \\
Forrest classification & \\
Ia & $7(16.3 \%)$ \\
Ib & $18(41.9 \%)$ \\
IIa & $18(41.9 \%)$ \\
Atrophic gastritis & \\
Closed type & $6(14.0 \%)$ \\
Open type & $31(72.1 \%)$ \\
Hemostatic method & \\
Soft coagulation & $42(97.7 \%)$ \\
Hemoclips & $1(2.3 \%)$ \\
Operator of hemostasis & \\
Trainees & $27(62.8 \%)$ \\
Specialists & $16(37.2 \%)$ \\
\hline
\end{tabular}

Group A: patients taking antithrombotic agents who underwent emergency endoscopic hemostasis for nonvariceal upper gastrointestinal bleeding and resumed antithrombotic medication without a cessation period.

deviation, and Student's $t$-test was used to determine differences between the two groups. Numerical data for a skewed distribution were expressed as median interquartile range, and the Mann-Whitney $U$-test was used. Levels of significance for all comparisons made were reported, whether significant or not, with $P$ values or confidence intervals. A $P$ value $<0.05$ was considered to be statistically significant for each test. All statistical analyses were performed with JMP, version 13.0.0 (SAS Institute Inc., Cary, NC, USA).

\section{Results}

3.1. Clinical Characteristics of the Prospective Cohort. Forty-three patients who resumed antithrombotic agents without cessation periods were allocated to group A. The characteristics of patients in group A are listed in Table 1. The mean age of the patients was 77.8 years, and $28 \mathrm{pa}$ tients $(65.1 \%)$ were males. The rate of $H$. pylori infection was $46.5 \%$. Cardiovascular disease was the most common comorbidity; it had been diagnosed in 23 patients.

Table 2 summarizes the details concerning antithrombotic agents among the patients. Thirty-two patients (74.4\%) took a single antithrombotic agent, and 11 patients (25.6\%) took multiple antithrombotic agents. The most common antiplatelet agent was aspirin (17 patients), and edoxaban (six patients), one of the direct oral anticoagulants
(DOACs), was the most frequently prescribed anticoagulant agent.

3.2. Endoscopic Findings and Clinical Outcome of the Prospective Cohort. The endoscopic findings of patients with nonvariceal UGIB are shown in Table 3 . Of the 41 patients with peptic ulcer disease, 34 patients complained of bleeding gastric ulcers, among whom multiple gastric ulcers were found in 15 patients. A large ulcer (>11 $\mathrm{mm}$ in diameter) was found in 21 patients, and spurting gastroduodenal bleeding was endoscopically identified in seven patients. To achieve endoscopic hemostasis, the soft coagulation method was applied to all but one patient, and 27 procedures (62.8\%) were performed by trainees. Consequently, endoscopic hemostasis was successfully carried out in all of the 43 patients.

The mean hemoglobin level on admission was $8.0 \pm 2.6 \mathrm{~g} /$ $\mathrm{dL}$, and blood transfusion was required with a mean volume of 3.2 units. After endoscopic hemostasis, all patients were treated with fasting and hospitalized for a mean period of $2.2 \pm 0.7 \mathrm{~d}$ and $9.1 \pm 5.5 \mathrm{~d}$, respectively. Rebleeding occurred in three patients (7.0\%); however, neither major thromboembolic events nor fatalities were recorded during the follow-up period.

\subsection{Comparison of Clinical Outcomes between Groups A and B} by Propensity Score Matching. Table 4 compares the clinical characteristics between groups A and B before and after propensity score matching. Before propensity score matching, the mean age $(77.8 \pm 9.1$ years in group A versus $72.8 \pm 12.4$ years in group $\mathrm{B}, P=0.004), H$. pylori infection $(46.5 \%$ versus $69.5 \%, P=0.007)$, antiplatelet agents $(69.8 \%$ versus $86.4 \%, P=0.02)$, and anticoagulant agents $(39.5 \%$ versus $21.4 \%, P=0.03)$ were significantly different between the two groups. Propensity score matching subsequently created 40 matched pairs in the present study.

Table 5 compares the treatment outcomes after propensity score matching between the two groups. Because group B comprised a retrospective cohort of patients who transiently discontinued antithrombotic agents after emergency endoscopic hemostasis, the cessation period of antithrombotic agents was significantly shorter in group A than in group B ( 0 versus $6.5 \mathrm{~d} ; P<0.001)$. Although the soft coagulation method was more frequently applied in group A than in group B $(97.5 \%$ versus $60.0 \%, P<0.001)$, endoscopic hemostasis was successfully achieved in all patients of both groups. The hospitalization periods were significantly shorter in group A than in group B $(8.6 \pm 5.2$ versus $14.4 \pm 7.1 \mathrm{~d} ; P<0.001)$.

Rebleeding within one month occurred in 2 (5.0\%) of 40 patients in group A and $6(15.0 \%)$ of 40 patients in group B. The treatment difference was calculated as $10.0 \%$ (95.0\% CI: -4.2 to 23.2 ), within an a priori $-10 \%$ noninferiority margin. Therefore, the rebleeding risk in the noncessation method of antithrombotic agents after emergency endoscopic hemostasis was not considered to be inferior to the transient discontinuation method in 
TABLE 4: Characteristics of patients before and after propensity score matching in groups A and B.

\begin{tabular}{|c|c|c|c|c|}
\hline & \multicolumn{4}{|c|}{ Before propensity score matching } \\
\hline & Group A & Group B & $P$ value & Standardized differences \\
\hline Number of patients $(\mathrm{N})$ & 43 & 154 & & \\
\hline Age (years) & $77.8 \pm 9.1$ & $72.8 \pm 12.4$ & 0.004 & 0.47 \\
\hline Gender, male & $28(65.1 \%)$ & $108(70.1 \%)$ & 0.58 & 0.11 \\
\hline Helicobacter pylori infection & $20(46.5 \%)$ & $107(69.5 \%)$ & 0.007 & 0.48 \\
\hline Number of antithrombotics, multiple & $11(25.6 \%)$ & $38(24.7 \%)$ & 1.00 & 0.02 \\
\hline Antiplatelet agents & $30(69.8 \%)$ & $133(86.4 \%)$ & 0.02 & 0.41 \\
\hline Anticoagulant agents & $17(39.5 \%)$ & $33(21.4 \%)$ & 0.03 & 0.40 \\
\hline Location of ulcer, gastric & $34(79.1 \%)$ & $108(84.4 \%)$ & 1.00 & 0.14 \\
\hline Number of ulcers, single & $28(65.1 \%)$ & $80(62.5 \%)$ & 0.86 & 0.05 \\
\hline \multirow[t]{3}{*}{ Size of ulcer, $>11 \mathrm{~mm}$} & $21(48.8 \%)$ & $83(64.8 \%)$ & 0.07 & 0.33 \\
\hline & \multicolumn{4}{|c|}{ After propensity score matching } \\
\hline & Group A & Group B & $P$ value & Standardized differences \\
\hline Number of patients $(\mathrm{N})$ & 40 & 40 & & \\
\hline Age (years) & $77.1 \pm 8.9$ & $78.2 \pm 8.0$ & 0.55 & 0.13 \\
\hline Gender, male & $26(65.0 \%)$ & $24(60.0 \%)$ & 0.82 & 0.10 \\
\hline Helicobacter pylori infection & $19(47,5 \%)$ & $20(50.0 \%)$ & 1.00 & 0.05 \\
\hline Number of antithrombotics, multiple & $11(27.5 \%)$ & $12(30.0 \%)$ & 1.00 & 0.05 \\
\hline Antiplatelet agents & $30(75.0 \%)$ & $31(77.5 \%)$ & 1.00 & 0.06 \\
\hline Anticoagulant agents & $14(35.0 \%)$ & $12(30.0 \%)$ & 0.81 & 0.11 \\
\hline Location of ulcer, gastric & $33(82.5 \%)$ & $36(90.0 \%)$ & 0.52 & 0.22 \\
\hline Number of ulcers, single & $26(65.0 \%)$ & $26(65.0 \%)$ & 1.00 & 0.00 \\
\hline Size of ulcer, $>11 \mathrm{~mm}$ & $21(52.5 \%)$ & $22(55.0 \%)$ & 1.00 & 0.05 \\
\hline
\end{tabular}

Group A: patients taking antithrombotic agents who underwent emergency endoscopic hemostasis for nonvariceal upper gastrointestinal bleeding and resumed antithrombotic medication without a cessation period; group B: patients taking antithrombotic agents who underwent emergency endoscopic hemostasis for nonvariceal upper gastrointestinal bleeding for whom antithrombotic medication was transiently discontinued.

TABLE 5: Comparison of treatment outcome between groups A and B after propensity score matching.

\begin{tabular}{lccc}
\hline & Group A & Group B & $P$ value \\
\hline Number of patients (N) & 40 & 40 & \\
Cessation period (day) & $0(0-0)$ & 6.5 & \\
Hemoglobin on admission (g/ & $8.1 \pm 2.6$ & $7.6 \pm 1.7$ & 0.31 \\
dL) & $3.0 \pm 2.6$ & $2.6 \pm 2.9$ & 0.58 \\
Transfusion volume (U) & $40(100 \%)$ & $40(100 \%)$ & 1.00 \\
Endoscopic hemostasis & & & $<0.001$ \\
Main modality of hemostasis & 39 & $24(60.0 \%)$ & \\
Soft coagulation & $(97.5 \%)$ & $14(35.0 \%)$ & \\
Hemoclips & $1(2.5 \%)$ & 14001 \\
$\quad$ Absolute ethanol injection & $0(0 \%)$ & $2(5 \%)$ & \\
Operator of hemostasis & & & 0.82 \\
$\quad$ Trainees & 24 & $26(65.0 \%)$ & \\
Specialists & $(60.0 \%)$ & & \\
Hospitalization periods (day) & $8.6 \pm 5.2$ & $14.4 \pm 7.1$ & $<0.001$ \\
\hline
\end{tabular}

${ }^{a}$ Median (interquartile range). Group A: patients taking antithrombotic agents who underwent emergency endoscopic hemostasis for nonvariceal upper gastrointestinal bleeding and resumed antithrombotic medication without a cessation period; group B: patients taking antithrombotic agents who underwent emergency endoscopic hemostasis for nonvariceal upper gastrointestinal bleeding for whom antithrombotic medication was transiently discontinued.

patients with nonvariceal UGIB (Figure 1). Neither thromboembolic events nor fatality occurred during the follow-up period.

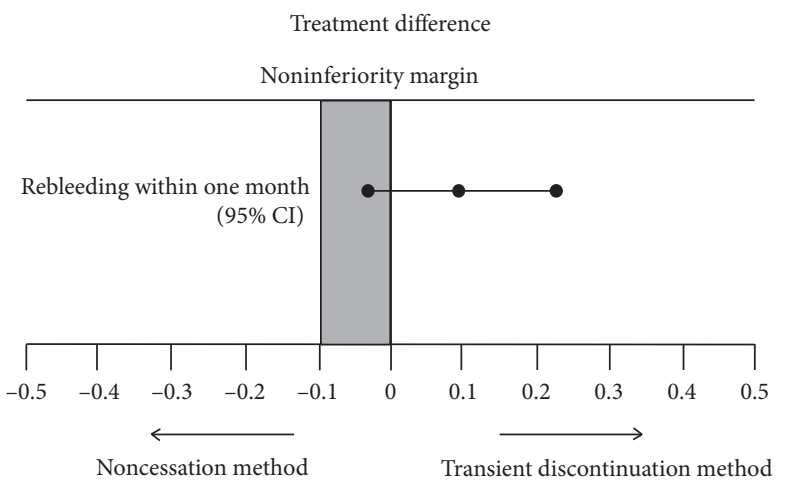

FIGURE 1: Difference in rebleeding rate within one month in patients taking antithrombotic agents who underwent emergency endoscopic hemostasis for nonvariceal upper gastrointestinal bleeding and resumed antithrombotic medication without a cessation period (group A) or transiently discontinued the medication (group B).

\section{Discussion}

In this super-aging society, both antiplatelet and anticoagulant agents have been increasingly used for the prophylaxis and treatment of vascular disease, especially cerebrovascular and cardiovascular disease. However, this practice has also led to an increase in the incidence of and morbidity associated with UGIB [25-28]. In addition, because the combination therapy of antithrombotic agents has become more prevalent [11] and because these medications may be associated with a higher risk of UGIB [29], medical 
management in patients with UGIB on antithrombotic agents has been a matter of recent debate.

The JGES guideline published in 2012 drastically changed the management of gastroenterological endoscopy in patients undergoing antithrombotic treatment [14]. The guideline was based on the fact that the clinical outcomes of thromboembolic events caused by the transient discontinuation of antithrombotic agents during the periendoscopic period were more severe compared with those of bleeding events among patients treated with the medication [30]. In fact, we also managed two patients who developed severe thromboembolic events after cessation of antithrombotic agents because of UGIB [11]. In contrast, repeat rebleeding in patients with UGIB after endoscopic hemostasis is troublesome for endoscopists, and some reports suggested a possible higher risk of rebleeding in patients with UGIB on antithrombotic agents [31, 32]. Furthermore, the clinical management of antithrombotic agents after invasive gastrointestinal endoscopy remains uncertain. We therefore compared the risk of rebleeding and safety of nonvariceal UGIB between patients on antithrombotic agents with our noncessation method and those treated in accordance with the JGES guidelines.

In the present study, the overall rebleeding rate within a month was $7.0 \%$ in patients with nonvariceal UGIB who continued antithrombotic agents after endoscopic hemostasis. The rebleeding rate in our previous cohort, classified as group B in the present study, in whom antithrombotic agents were transiently discontinued, was 9.7\% (15/154 patients) [11]. That value was almost equivalent to the rebleeding rates in other previous studies $(7.0 \%$ to $17.8 \%)$ [32-34]. Consequently, the difference in the rebleeding rate between groups $\mathrm{A}$ and $\mathrm{B}$ after propensity score matching was demonstrated to be within the noninferiority margin. We therefore considered that our noncessation method of antithrombotic agents did not lead to an increased risk of rebleeding after emergency endoscopic hemostasis in patients with nonvariceal UGIB.

The rebleeding rate after endoscopic hemostasis can be influenced by the hemostatic procedures applied. In the present study, the rate of rebleeding was numerically but not significantly higher in group B than in group A after propensity score matching. Because the soft coagulation method is a useful hemostatic procedure during ESD [20, 35-38], ESD has also become more prevalent for endoscopic hemostasis in patients with nonvariceal UGIB in Japan [16, 39]. In addition, because our previous study demonstrated that soft coagulation was significantly superior to hemoclipping for endoscopic hemostasis in such patients [16], we have preferentially chosen the soft coagulation method over hemoclipping. As a result, soft coagulation was more frequently applied in group A than in group B (97.5\% in group A versus $60.0 \%$ in group $B, P<0.001)$. However, it seems unlikely that the difference in the procedure affected the rebleeding rate because the rebleeding rates by hemoclipping and by soft coagulation were similar in group B $(14.3 \%$ versus $16.7 \%$, respectively).

No patient experienced major complications (bleeding or thromboembolism) in the present study. However, we previously reported adverse events in two patients who experienced cerebral infarction during the cessation period of antithrombotic agents [11]. Another report also described six thromboembolic events occurring in 36 patients with nonvariceal UGIB who discontinued warfarin as well as one myocardial infarction in 41 patients with nonvariceal UGIB who discontinued aspirin [33]. A meta-analysis also demonstrated that the resumption of warfarin following interruption because of GI bleeding was significantly associated with a reduction in thromboembolic events (hazard ratio [HR]: 0.68, 95\% CI: 0.52-0.88) and in mortality (HR: 0.76, 95\% CI: 0.66-0.88) [40]. These results indicated that we should be strictly aware of the potential for thromboembolic events by the interruption of antithrombotic agents after UGIB.

In addition, such unfavorable outcome can be also caused by hemodynamic instability due to UGIB. Actually, a cohort study of emergency admissions for nonmalignant UGIB reported that $8.9 \%$ of deaths after 30 days of hospitalization were due to ischemic heart disease and the cardiovascular age-standardized mortality rate following UGIB was high [41]. Another study examining the risk of rebleeding and in-hospital mortality after UGIB demonstrated that $1.5 \%$ of patients with UGIB died of thromboembolism, and poor performance status (PS; $\geq 3$ ) and tachycardia (>100/min) were significantly associated with mortality [42]. Considering the higher risk of thromboembolism in patients with UBIG, efforts to decrease the risk seem indispensable, especially in patients with antithrombotic agents. Together with the fact that no major bleeding adverse events occurred in group A of the present study, the noncessation method after emergency endoscopic hemostasis seems to be appropriate for the management of patients with nonvariceal UGIB on antithrombotic treatment.

While no significant difference in rebleeding and thromboembolic adverse events were identified in comparing groups $\mathrm{A}$ and $\mathrm{B}$, the hospitalization period was significantly shorter in the former than in the latter. Such a difference might be partly attributed to the numerically higher rate of rebleeding in group B. However, this apparent advantage of the noncessation method of antithrombotic agents after endoscopic hemostasis should be considered cautiously because warfarin requires close monitoring of the prothrombin time and international normalized ratio when resuming. Warfarin was used more frequently as anticoagulant therapy in group B (nine of nine patients, 100\%) than in group A (two of eleven patients, 18.2\%). Because DOACs have recently become the mainstay of anticoagulant therapy, further study is necessary to determine the difference in hospitalization period among patients with nonvariceal UGIB taking DOACs.

Our present study has some limitations. First, the number of subjects in the prospective cohort was small because of the study design. Therefore, the low rebleeding rate in the prospective cohort can be a statistical type II error. Second, a considerable number of endoscopic hemostases in both groups were performed by trainees. While all procedures of endoscopic hemostasis by trainees were performed 
under the guidance of specialists, insufficient hemostatic techniques of the trainees could have caused the numerical difference in the rebleeding rate between the two groups. Third, the retrospective cohort of our previous study was used to compare clinical outcome and adverse events between patients with and without a cessation period of antithrombotic agents after endoscopic hemostasis. While propensity score matching analysis was applied to minimize the difference in clinical characteristics between the two groups, the results of the present study should be interpreted with caution because of the different types of cohort between the groups. Further prospective study with a larger number of subjects should be done to validate the results of the present study.

\section{Conclusions}

This multicenter pilot study demonstrated that continuing antithrombotic agents without a cessation period was acceptable after emergency endoscopic hemostasis in patients with nonvariceal UGIB and could shorten the hospitalization period.

\section{Abbreviations}

DOACs: Direct oral anticoagulants

ESD: Endoscopic submucosal dissection

JGES: Japan Gastroenterological Endoscopy Society

UGIB: Upper gastrointestinal bleeding.

\section{Data Availability}

The datasets used and/or analyzed during the current study are available from the corresponding author upon reasonable request.

\section{Ethical Approval}

This study was conducted in accordance with the Declaration of Helsinki and the guidelines of the Consolidated Standards of Reporting Trials (CONSORT). The study protocol and the consent procedure were approved by the Ethics Review Committee of the National Hospital Organization Ureshino Medical Center.

\section{Consent}

Written informed consent was obtained after endoscopic hemostasis from patients who met the inclusion criteria.

\section{Disclosure}

An earlier version of this manuscript has been presented as Preprint in Research Square (https://www.researchsquare. com/article/rs-48832/v1). This study was registered with the University Hospital Medical Information Network (UMIN) Clinical Trials Registry (UMIN000030879) on 22 January 2018.

\section{Conflicts of Interest}

The authors declare that they have no conflicts of interest.

\section{Authors' Contributions}

Daisuke Yamaguchi designed the research, designed the work, and analyzed the data; Daisuke Yamaguchi, Naoyuki Tominaga, Koichi Miyahara, Nanae Tsuruoka, Yasuhisa Sakata, Yuki Takeuchi, Takuya Matsunaga, Hidenori Hidaka, Takashi Akutagawa, Takahiro Noda, Shinichi Ogata, and Seiji Tsunada performed endoscopic hemostasis, acquired the patient data, and made substantial contributions to the conception; DY and Motohiro Esaki wrote the paper; ME was a major contributor in writing the manuscript. All authors have read and approved the submitted version and approved the final manuscript. All authors have agreed to both be personally accountable for the authors' own contributions and ensure that questions related to the accuracy or integrity of any part of the work, even ones in which the authors were not personally involved, are appropriately investigated and resolved, and the resolution is documented in the literature.

\section{Acknowledgments}

The authors thank Dr. Ayako Takamori, Clinical Research Center, Saga University Hospital, for statistical advice. The authors also thank Andrea Baird, M.D., from Edanz Group (https://en-author-services.edanzgroup.com/), for editing a draft of this manuscript.

\section{Supplementary Materials}

Supplementary Table 1: characteristics of patients in group B. Supplementary Table 2: types of antithrombotic agents taken by patients in group B. Supplementary Table 3: endoscopic findings and hemostatic methods in patients in group B. (Supplementary Materials)

\section{References}

[1] D. Yamaguchi, Y. Sakata, H. Yoshida et al., "Effectiveness of endoscopic hemostasis with soft coagulation for non-variceal upper gastrointestinal bleeding over a 12-year period," Digestion, vol. 95, no. 4, pp. 319-326, 2017.

[2] H. Ootani, R. Iwakiri, R. Shimoda et al., "Role of Helicobacter pylori infection and nonsteroidal anti-inflammatory drug use in bleeding peptic ulcers in Japan," Journal of Gastroenterology, vol. 41, no. 1, pp. 41-46, 2006.

[3] M. Fujishiro, M. Iguchi, N. Kakushima et al., "Guidelines for endoscopic management of non-variceal upper gastrointestinal bleeding," Digestive Endoscopy, vol. 28, no. 4, pp. 363-378, 2016.

[4] F. Baracat, E. Moura, W. Bernardo et al., "Endoscopic hemostasis for peptic ulcer bleeding: systematic review and meta-analyses of randomized controlled trials," Surgical Endoscopy, vol. 30, no. 6, pp. 2155-2168, 2016.

[5] S. Mujtaba, S. Chawla, and J. F. Massaad, "Diagnosis and management of non-variceal gastrointestinal hemorrhage: a 
review of current guidelines and future perspectives," Journal of Clinical Medicine, vol. 9, p. E402, 2020.

[6] M. Nakayama, R. Iwakiri, M. Hara et al., "Low-dose aspirin is a prominent cause of bleeding ulcers in patients who underwent emergency endoscopy," Journal of Gastroenterology, vol. 44, no. 9, pp. 912-918, 2009.

[7] K. Nishikawa, T. Sugiyama, M. Kato et al., "Non-Helicobacter pylori and non-NSAID peptic ulcer disease in the Japanese population," European Journal of Gastroenterology \& Hepatology, vol. 12, no. 6, pp. 635-640, 2000.

[8] S. P. Johnsen, H. T. Sørensen, L. Mellemkjoer et al., "Hospitalisation for upper gastrointestinal bleeding associated with use of oral anticoagulants," Thrombosis and Haemostasis, vol. 86, pp. 563-568, 2001.

[9] T. Higuchi, R. Iwakiri, M. Hara et al., "Low-dose aspirin and comorbidities are significantly related to bleeding peptic ulcers in elderly patients compared with nonelderly patients in Japan," Internal Medicine, vol. 53, no. 5, pp. 367-373, 2014.

[10] D. Yamaguchi, Y. Sakata, N. Tsuruoka et al., "Upper Gastrointestinal bleeding in Japanese patients prescribed antithrombotic drugs: differences in trends over time," Hepato-Gastroenterology, vol. 61, pp. 1055-1062, 2014.

[11] D. Yamaguchi, Y. Sakata, N. Tsuruoka et al., "Characteristics of patients with non-variceal upper gastrointestinal bleeding taking antithrombotic agents," Digestive Endoscopy, vol. 27, no. 1, pp. 30-36, 2015.

[12] S. Ono, M. Fujishiro, H. Kanzaki et al., "Conflicting clinical environment about the management of antithrombotic agents during the periendoscopic period in Japan," Journal of Gastroenterology and Hepatology, vol. 26, pp. 1434-1440, 2011.

[13] Y. Sanomura, S. Oka, S. Tanaka et al., "Continued use of lowdose aspirin does not increase the risk of bleeding during or after endoscopic submucosal dissection for early gastric cancer," Gastric Cancer, vol. 17, no. 3, pp. 489-496, 2014.

[14] K. Fujimoto, M. Fujishiro, M. Kato et al., "Guidelines for gastroenterological endoscopy in patients undergoing antithrombotic treatment," Digestive Endoscopy, vol. 26, no. 1, pp. 1-14, 2014.

[15] K. Ogoshi, E. Kaneko, M. Tada et al., "Japan Gastroenterological Endoscopy Society Risk Management Committee. Use of anticoagulants and antiplatelet agents during endoscopic procedures," Gastroenterol Endos, vol. 47, pp. 2691-2695, 2005, (in Japanese.).

[16] S. Arima, Y. Sakata, S. Ogata et al., "Evaluation of hemostasis with soft coagulation using endoscopic hemostatic forceps in comparison with metallic hemoclips for bleeding gastric ulcers: a prospective, randomized trial," Journal of Gastroenterology, vol. 45, no. 5, pp. 501-505, 2010.

[17] R. Shimoda, R. Iwakiri, H. Sakata et al., "Evaluation of endoscopic hemostasis with metallic hemoclips for bleeding gastric ulcer: comparison with endoscopic injection of absolute ethanol in a prospective, randomized study," American Journal of Gastroenterology, vol. 98, no. 10, pp. 2198-2202, 2003.

[18] R. Shimoda, R. Iwakiri, H. Sakata et al., "Endoscopic hemostasis with metallic hemoclips for iatrogenic MalloryWeiss tear caused by endoscopic examination," Digestive Endoscopy, vol. 21, no. 1, pp. 20-23, 2009.

[19] Y. Tsuji, K. Ohata, M. Sekiguchi et al., "An effective training system for endoscopic submucosal dissection of gastric neoplasm," Endoscopy, vol. 43, no. 12, pp. 1033-1038, 2011.

[20] H. Ono, K. Yao, M. Fujishiro et al., "Guidelines for endoscopic submucosal dissection and endoscopic mucosal resection for early gastric cancer," Digestive Endoscopy, vol. 28, no. 1, pp. 3-15, 2016.

[21] S.-J. Cho, I. Choi, C. Kim et al., "Aspirin use and bleeding risk after endoscopic submucosal dissection in patients with gastric neoplasms," Endoscopy, vol. 44, no. 2, pp. 114-121, 2012.

[22] C. Sato, K. Hirasawa, R. Koh et al., "Postoperative bleeding in patients on antithrombotic therapy after gastric endoscopic submucosal dissection," World Journal of Gastroenterology, vol. 23, no. 30, pp. 5557-5566, 2017.

[23] T. Furuhata, M. Kaise, S. Hoteya et al., "Postoperative bleeding after gastric endoscopic submucosal dissection in patients receiving antithrombotic therapy," Gastric Cancer, vol. 20, no. 1, pp. 207-214, 2017.

[24] R. B. D’Agostino Jr., "Propensity score methods for bias reduction in the comparison of a treatment to a nonrandomized control group," Statistics in Medicine, vol. 17, pp. 2265-2281, 1998.

[25] S. D. Fihn, M. McDonell, D. Martin et al., "Risk factors for complications of chronic anticoagulation: a multicenter study," Annals of Internal Medicine, vol. 118, no. 7, pp. 511-520, 1993.

[26] A. S. Taha, W. J. Angerson, R. P. Knill-Jones, and O. Blatchford, "Clinical outcome in upper gastrointestinal bleeding complicating low-dose aspirin and antithrombotic drugs," Alimentary Pharmacology \& Therapeutics, vol. 24, no. 4, pp. 633-636, 2006.

[27] N. Nagata, H. Yasunaga, H. Matsui et al., "Therapeutic endoscopy-related GI bleeding and thromboembolic events in patients using warfarin or direct oral anticoagulants: results from a large nationwide database analysis," Gut, vol. 67, no. 10, pp. 1805-1812, 2018.

[28] M. M. Brodie, J. C. Newman, T. Smith, and D. C. Rockey, "Severity of gastrointestinal bleeding in patients treated with direct-acting oral anticoagulants," The American Journal of Medicine, vol. 131, pp. 573.e9-573.e15, 2018.

[29] J. Hallas, M. Dall, A. Andries et al., "Use of single and combined antithrombotic therapy and risk of serious upper gastrointestinal bleeding: population based case-control study," BMJ, vol. 333, no. 7571, pp. 726-731, 2006.

[30] S. Ono, M. Fujishiro, S. Kodashima et al., "Evaluation of safety of endoscopic biopsy without cessation of antithrombotic agents in Japan," Journal of Gastroenterology, vol. 47, no. 7, pp. 770-774, 2012.

[31] P. García-Iglesias, A. Villoria, D. Suarez et al., "Meta-analysis: predictors of rebleeding after endoscopic treatment for bleeding peptic ulcer," Alimentary Pharmacology \& Therapeutics, vol. 34, no. 8, pp. 888-900, 2011.

[32] M. J. Hong, S.-Y. Lee, J. H. Kim et al., "Rebleeding after initial endoscopic hemostasis in peptic ulcer disease," Journal of Korean Medical Science, vol. 29, no. 10, pp. 1411-1415, 2014.

[33] J. K. Lee, H. W. Kang, S. G. Kim, J. S. Kim, and H. C. Jung, "Risks related with withholding and resuming anticoagulation in patients with non-variceal upper gastrointestinal bleeding while on warfarin therapy," International Journal of Clinical Practice, vol. 66, no. 1, pp. 64-68, 2012.

[34] Y. J. Han, J. M. Cha, J. H. Park et al., "Successful endoscopic hemostasis is a protective factor for rebleeding and mortality in patients with nonvariceal upper gastrointestinal bleeding," Digestive Diseases and Sciences, vol. 61, no. 7, pp. 2011-2018, 2016.

[35] H. Ono, H. Kondo, T. Gotoda et al., "Endoscopic mucosal resection for treatment of early gastric cancer," Gut, vol. 48, no. 2, pp. 225-229, 2001. 
[36] K. Mannen, S. Tsunada, M. Hara et al., "Risk factors for complications of endoscopic submucosal dissection in gastric tumors: analysis of 478 lesions," Journal of Gastroenterology, vol. 45 , no. 1 , pp. $30-36,2010$.

[37] K. Miyahara, R. Iwakiri, R. Shimoda et al., "Perforation and postoperative bleeding of endoscopic submucosal dissection in gastric tumors: analysis of 1,190 lesions in low- and highvolume centers in Saga, Japan," Digestion, vol. 86, no. 3, pp. 273-280, 2012.

[38] K. Yamanouchi, S. Ogata, Y. Sakata et al., "Effect of additional surgery after noncurative endoscopic submucosal dissection for early gastric cancer," Endoscopy International Open, vol. 4, pp. E24-E29, 2016.

[39] S. Nagata, S. Kimura, H. Ogoshi, and T. Hidaka, "Endoscopic hemostasis of gastric ulcer bleeding by hemostatic forceps coagulation," Digestive Endoscopy, vol. 22, no. 1, pp. S22-S25, 2010.

[40] C. Chai-Adisaksopha, C. Hillis, M. Monreal, D. Witt, and M. Crowther, "Thromboembolic events, recurrent bleeding and mortality after resuming anticoagulant following gastrointestinal bleeding," Thrombosis and Haemostasis, vol. 114, no. 10, pp. 819-825, 2015.

[41] J. Rees, F. Evison, J. Mytton, P. Patel, and N. Trudgill, “The outcomes of emergency hospital admissions with nonmalignant upper gastrointestinal bleeding in England between 2003 and 2015," Endoscopy, 2021.

[42] S. Fukuda, Y. Shimodaira, K. Watanabe et al., "Risks for rebleeding and in-hospital mortality after gastrointestinal bleeding in a tertiary referral center in Japan," Digestion, vol. 101, no. 1, pp. 31-37, 2020. 